\title{
Highly Efficient Synthesis of Conformationally Fixed Bicyclo[3.1.0]hexyl Nucleosides with an Ethenyl Group at C3'-Position as Potential Antiviral Agents
}

\author{
Seong Jin Kim, ${ }^{\mathrm{a}}$ Youngwoo Woo, ${ }^{\mathrm{a}}$ Ah-Young Park, ${ }^{\mathrm{a}}$ Hye Rim Kim, Sujin Son, \\ Hwi Young Yun, Pusoon Chun, ${ }^{\dagger}$ and Hyung Ryong Moon* \\ College of Pharmacy and Research Institute for Drug Development, Pusan National University, \\ Busan 609-735, Korea. *E-mail: mhr108@pusan.ac.kr \\ ${ }^{\dagger}$ College of Pharmacy, Inje University, Gimhae, Gyeongnam 621-749, Korea \\ Received April 20, 2014, Accepted May 7, 2014
}

\begin{abstract}
Synthesis of north-5'-methylbicyclo[3.1.0]hexyl adenine and hypoxanthine nucleosides with an ethenyl group at C3' position was successfully achieved by a highly facile method. Methylbicyclo[3.1.0] hexanone ( \pm )-7 with three contiguous chiral centers and its epimer $( \pm)-6$ was remarkably simply constructed only by four steps involving a carbenoid insertion reaction in the presence of rhodium (II) acetate dimer as a metal catalyst, giving a correct relative stereochemistry of the generated three chiral centers. Due to steric hindrance from the concave face of the bicyclo[3.1.0] hexanone system, a Grignard reaction of $( \pm)-7$ with ethenylmagnesium bromide showed exclusive diastereoselectivity towards the b-face. The Grignard reaction chemoselectively proceeded without reacting with ester functionality. Coupling reaction of glycosyl donor ( \pm )-11 with 6-chloropurine nucleobase afforded only the desired $N^{9}$-alkylated nucleoside without the formation of $N^{7}$-regioisomer. By the conventional method, 6-chloro group was converted into 6-amino and 6-hydroxy groups to give the desired adenine and hypoxanthine bicyclo[3.1.0] hexyl carbanucleosides with 3'-ethenyl group, respectively.
\end{abstract}

Key Words : 5-Methylbicyclo[3.1.0]hexanone, Carbenoid cycloaddition, 3'-Ethenyl, North conformation, Mitsunobu reaction

\section{Introduction}

Although the carbocyclic nucleosides ${ }^{1-3}$ in which the furanose oxygen of natural nucleosides is replaced by a methylene group are chemically more stable due to the absence of a true glycosidic bond, they adopts a different conformation from that of normal nucleosides with a tetrahydrofuran ring because of conversion of the furanose ring into a cyclopentane ring. Nucleosides should be phosphorylated and converted to their triphosphate forms by kinases to become bioactive antimetabolites, which act as DNA or RNA chain terminators and/or competitive inhibitors of DNA/RNA polymerases. Normal nucleosides adopt a northern or southern conformation as one of the most stable conformations by the interplay of important interactions resulting from anomeric and gauche effects. ${ }^{4,5}$ Kinases associated with the metabolism of nucleosides into their triphosphates prefer nucleosides with a northern or southern conformation as their substrates. This fact suggests that the rigid nucleosides with a northern or southern conformation are able to be more easily converted into triphosphates by kinases and would become even more potent antimetabolites. A bicyclo[3.1.0] hexane scaffold in which a cyclopentane ring is fused with a cyclopropane ring is reported to adopt a rigid south or north conformation. ${ }^{6}$ Therefore, rigid carbocyclic nucleosides built on a bicyclo[3.1.0]hexane template ${ }^{7-11}$ have received considerable attention as potential anticancer and

\footnotetext{
${ }^{\mathrm{a}}$ These authors contributed equally to this work.
}
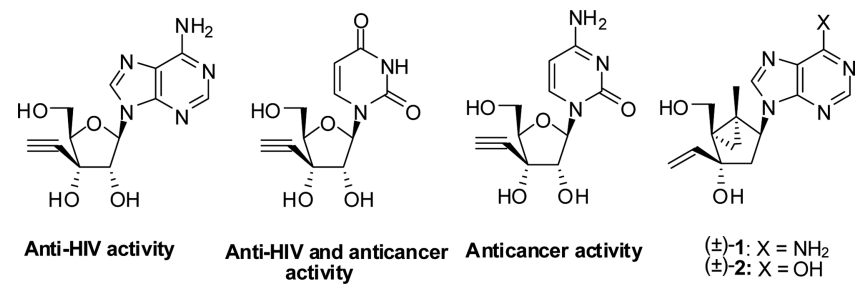

Figure 1. Nucleosides with a 3 '-substituent and target compounds, $( \pm)-\mathbf{1}$ and $( \pm)$-2.

antiviral agents.

Introduction of unsaturated substituents such as an acetylenyl or azido group into $3^{\prime}$ or $4^{\prime}$ position of normal nucleosides has been conducted to induce potent anticancer and antiviral activities (Figure 1). ${ }^{12,13}$ Particularly, replacement of a 3'-hydrogen atom by an acetylenyl or vinyl group converts the secondary 3'-hydroxyl group into tertiary one, indicating that this change might induce a decreased nucleophilicity of 3'-hydroxyl group towards the phosphoester of nucleoside triphosphate during DNA or RNA chain elongation process due to its reduced nucleophilicity by an electronic effect and steric hindrance. Therefore, nucleosides with a 3 '-substituent might act as a DNA terminator, exerting anticancer and antiviral activity.

Therefore, it was very interesting to synthesize north-fixed bicyclo[3.1.0] hexyl nucleosides with a 3'-ethenyl substituent as potential anticancer and antiviral agents. According to the methodology developed by the author et al.,,14 a desired 5- 
methylbicyclo[3.1.0]hexanone template $( \pm)$-7 was successfully synthesized with remarkable easiness using an 1,2addition reaction and a carbenoid cycloaddition, and a highly diastereo- and chemo-selective Grignard reaction of the ketone $( \pm)-7$ with ethenylmagnesium bromide was observed.

\section{Experimental}

Melting Points are Uncorrected. ${ }^{1} \mathrm{H}$ and ${ }^{13} \mathrm{C}$ NMR spectra were recorded on Varian Unity INOVA 400 and Varian Unity AS 500 instruments. Chemical shifts are reported with reference to the respective residual solvent or deuteriated peaks $\left(\delta_{\mathrm{H}} 3.30\right.$ and $\delta_{\mathrm{C}} 49.0$ for $\mathrm{CD}_{3} \mathrm{OD}, \delta_{\mathrm{H}} 7.27$ and $\delta_{\mathrm{C}} 77.0$ for $\left.\mathrm{CDCl}_{3}\right)$. Coupling constants are reported in hertz. The abbreviations used are as follows: s (singlet), $d$ (doublet), $\mathrm{m}$ (multiplet), t (triplet), dd (doublet of doublet), br s (broad singlet). All the reactions described below were performed under argon or nitrogen atmosphere and monitored by TLC. All anhydrous solvents were distilled over $\mathrm{CaH}_{2}$ or $\mathrm{Na}$ / benzophenone prior to use.

( \pm )-Ethyl 5-[(tert-butyldiphenylsilyl)oxy]-6-methyl-3oxohept-6-enoate (( \pm )-4). A stirred solution of LDA (2.0 M in THF, $131 \mathrm{~mL}, 262.0 \mathrm{mmol})$ in anhydrous THF $(350 \mathrm{~mL})$ was treated dropwise with ethyl acetoacetate $(13.3 \mathrm{~mL}$, $104.6 \mathrm{mmol}$ ) at $0{ }^{\circ} \mathrm{C}$. After $20 \mathrm{~min}$ the reation mixture was cooled to $-78{ }^{\circ} \mathrm{C}$, and methacrolein $(10 \mathrm{~mL}, 148.6 \mathrm{mmol})$ was added while stirring continued for $20 \mathrm{~min}$ at $-78{ }^{\circ} \mathrm{C}$. Following quenching with saturated $\mathrm{NH}_{4} \mathrm{Cl}(40 \mathrm{~mL})$, the reaction mixture was extracted with diethyl ether $(3 \times 150$ $\mathrm{mL})$. The organic extracts were combined, dried over $\mathrm{MgSO}_{4}$, and concentrated under vacuum to give a crude product $( \pm)$ 1 (29.96 g), which was used in the next step without further purification. A stirred solution of the crude product $( \pm)-\mathbf{1}$ and imidazole (11.76 g, $172.68 \mathrm{mmol}$ ) in methylene chloride $(250 \mathrm{~mL})$ was maintained at $0{ }^{\circ} \mathrm{C}$ and treated with TBDPSCl (22.5 mL, $86.34 \mathrm{mmol})$. After stirring for $6 \mathrm{~h}$ and allowing the reaction temperature to reach ambient temperature, water $(120 \mathrm{~mL})$ and methylene chloride $(250 \mathrm{~mL})$ were added. The organic layer was separated, washed with brine $(20 \mathrm{~mL})$, dried over $\mathrm{MgSO}_{4}$, filtered, and concentrated under reduced pressure. The resulting residue was purified by silica gel column chromatography using hexane and ethyl acetate $(18: 1)$ as the eluent to give $( \pm)-4(22.97 \mathrm{~g}, 67 \%)$, which existed in a rapid equilibrium with its enol tautomer, as a colorless oil: ${ }^{1} \mathrm{H} \mathrm{NMR}\left(500 \mathrm{MHz}, \mathrm{CDCl}_{3}\right) \delta 11.81(\mathrm{~s}, 1 \mathrm{H}$, enolic proton, minor), 7.70-7.35 (m, $20 \mathrm{H}, 4 \times \mathrm{Ar}$, major and minor), 4.89 (s, $1 \mathrm{H}, 2-\mathrm{H}$, minor), $4.84(\mathrm{~m}, 1 \mathrm{H}, \mathrm{C}=\mathrm{CH}$, major), 4.76-4.71 (m, $3 \mathrm{H}, \mathrm{C}=\mathrm{CH} H, \mathrm{C}=\mathrm{CH}_{2}$, major and minor), 4.63 (t, $1 \mathrm{H}, J=6.0 \mathrm{~Hz}, 5-\mathrm{CH}$, major), 4.50 (t, $1 \mathrm{H}, J$ $=6.5 \mathrm{~Hz}, 5-\mathrm{CH}$, minor), 4.20-4.13 (m, $4 \mathrm{H}, \mathrm{OCH}_{2} \mathrm{CH}_{3}$, major and minor), 3.30 (s, $2 \mathrm{H}, 2-\mathrm{CH}_{2}$, major), 2.74 (dd, $1 \mathrm{H}, J=$ 6.0, $14.5 \mathrm{~Hz}, 4-\mathrm{CHH}$, major), 2.70 (dd, $1 \mathrm{H}, J=6.0,15.0 \mathrm{~Hz}$, 4-CHH, major), 2.42 (dd, $1 \mathrm{H}, J=7.0,14.0 \mathrm{~Hz}, 4-\mathrm{CH}$, minor), 2.33 (dd, $1 \mathrm{H}, J=7.0,13.5 \mathrm{~Hz}, 4-\mathrm{CH} H$, minor), 1.72 (br s, $3 \mathrm{H}, 6-\mathrm{CH}_{3}$, minor), 1.69 (br s, $3 \mathrm{H}, 6-\mathrm{CH}_{3}$, major), 1.29 (t, $3 \mathrm{H}, J=7.0 \mathrm{~Hz}, \mathrm{OCH}_{2} \mathrm{CH}_{3}$, minor), 1.26 (t, $3 \mathrm{H}, J=$ $7.0 \mathrm{~Hz}, \mathrm{OCH}_{2} \mathrm{CH}_{3}$, major), 1.09 (s, $9 \mathrm{H}$, tert-butyl, major),
1.08 (s, $9 \mathrm{H}$, tert-butyl, minor); ${ }^{13} \mathrm{C} \mathrm{NMR}\left(125 \mathrm{MHz}, \mathrm{CDCl}_{3}\right)$ $\delta 200.37$ (major), 174.81 (minor), 172.37 (minor), 166.95 (major), 145.35 (minor), 145.14 (major), 136.00 (minor), 135.94 (major), 135.91 (minor), 135.89 (major), 133.91 (minor), 133.68 (major), 133.64 (minor), 133.30 (major), 129.77 (major), 129.70 (major), 129.56 (minor), 129.55 (minor), 127.59 (major), 127.45 (major), 127.42 (minor), 127.33 (minor), 112.61 (major), 112.49 (minor), 91.23 (minor), 74.97 (minor), 73.44 (major), 61.20 (major), 59.84 (minor), 50.07 (major), 49.53 (major), 42.57 (minor), 26.97 (major), 26.95 (minor), 19.33 (minor), 19.30 (major), 17.29 (major), 16.86 (minor), 14.24 (minor), 14.05 (major); LRMS $(\mathrm{FAB}+) m / z: 381(\mathrm{M}+\mathrm{H}-t-\mathrm{Bu})^{+}, 393(\mathrm{M}+\mathrm{H}-\mathrm{EtOH})^{+}, 439$ $(\mathrm{M}+\mathrm{H})^{+}, 461(\mathrm{M}+\mathrm{Na})^{+}$.

( \pm )-Ethyl 2-Diazo-5-[(tert-butyldiphenylsilyl)oxy]-6methyl-3-oxohept-6-enoate $(( \pm)-5)$. A stirred solution of $\beta$ keto ester $( \pm)-4(22.97 \mathrm{~g}, 52.37 \mathrm{mmol})$ and tosyl azide $(10.33 \mathrm{~g}, 52.38 \mathrm{mmol})$ in $\mathrm{CH}_{3} \mathrm{CN}(105 \mathrm{~mL})$ was treated with triethylamine $(14.59 \mathrm{~mL}, 104.68 \mathrm{mmol})$ at $0{ }^{\circ} \mathrm{C}$. After $2 \mathrm{~h}$ of stirring at $0{ }^{\circ} \mathrm{C}$, the reaction mixture was to reach room temperature and stirring was continued for $21 \mathrm{~h}$. Diethyl ether $(350 \mathrm{~mL})$ and $2 \mathrm{~N} \mathrm{NaOH}$ aqueous solution $(350 \mathrm{~mL})$ were added. After $10 \mathrm{~min}$ of stirring, the organic layer was separated, washed with brine $(30 \mathrm{~mL})$, dried over $\mathrm{MgSO}_{4}$, filtered, and concentrated under vacuum. The resulting residue was purified by silica gel column chromatography using hexane and ethyl acetate (11:1) as the eluent to give diazo compound $( \pm)-5(23.8 \mathrm{~g}, 98 \%)$ as a colorless oil: ${ }^{1} \mathrm{H}$ NMR (500 MHz, $\left.\mathrm{CDCl}_{3}\right) \delta 7.66-7.33(\mathrm{~m}, 10 \mathrm{H}, 2 \times \mathrm{Ar})$, $4.78\left(\mathrm{~m}, 1 \mathrm{H}, \mathrm{CH}_{3} \mathrm{C}=\mathrm{CHH}\right), 4.75(\mathrm{dd}, 1 \mathrm{H}, J=5.0,8.0 \mathrm{~Hz}, 5-$ $\mathrm{CH}), 4.71\left(\mathrm{~m}, 1 \mathrm{H}, \mathrm{CH}_{3} \mathrm{C}=\mathrm{CH} H\right), 4.24\left(\mathrm{~m}, 2 \mathrm{H}, \mathrm{OCH}_{2} \mathrm{CH}_{3}\right)$, $3.21(\mathrm{dd}, 1 \mathrm{H}, J=8.0,14.5 \mathrm{~Hz}, \mathrm{COCHH}), 2.97$ (dd, $1 \mathrm{H}, J=$ 5.0, $15.0 \mathrm{~Hz}, \mathrm{COCH} H), 1.74\left(\mathrm{~s}, 3 \mathrm{H}, \mathrm{CH}_{2}=\mathrm{CCH}_{3}\right), 1.31(\mathrm{t}, 3$ $\left.\mathrm{H}, J=7.3 \mathrm{~Hz}, \mathrm{OCH}_{2} \mathrm{CH}_{3}\right), 1.05$ (s, $9 \mathrm{H}$, tert-butyl); ${ }^{13} \mathrm{C}$ NMR $\left(125 \mathrm{MHz}, \mathrm{CDCl}_{3}\right) \delta 189.88,161.08,146.06,136.06$, $135.97,134.02,133.68,129.49,129.45,127.30,127.26$, 112.21, 74.02, 61.25, 46.76, 26.95, 19.34, 17.10, 14.32; LRMS $(\mathrm{FAB}+) m / z: 407(\mathrm{M}-t-\mathrm{Bu})^{+}, 465(\mathrm{M}+\mathrm{H})^{+}, 487(\mathrm{M}+\mathrm{Na})^{+}$.

$( \pm)-($ rel $)-(1 S, 4 S, 5 S)$-Ethyl 4-[(tert-butyldiphenylsilyl)oxy]-5-methyl-2-oxobicyclo[3.1.0]hexane-1-carboxylate $(( \pm)-6)$ and $( \pm)-($ rel $)-(1 S, 4 R, 5 S)-$ ethyl 4-[(tert-butyldiphenylsilyl)oxy]-5-methyl-2-oxobicyclo[3.1.0] hexane-1-carboxylate $(( \pm)-7)$. To a stirred solution of diazo compound $( \pm)-5$ $(23.8 \mathrm{~g}, 51.22 \mathrm{mmol})$ in benzene $(320 \mathrm{~mL})$ was added rhodium (II) acetate dimer ( $5 \mathrm{mg}, 0.01 \mathrm{mmol})$ at room temperature. After stirring for $24 \mathrm{~h}$, the reaction mixture was filtered through a pad of Celite, and washed with ethyl acetate. The organic solvent was concentrated under vacuum and the resulting residue was purified by silica gel column chromatography using hexane and ethyl acetate (10:1) as the eluent to give the desired bicyclic compound $( \pm)-7$ (8.05 g, $36 \%)$ and its C-4 epimer $( \pm)-6(11.55 \mathrm{~g}, 52 \%)$ as a colorless oil, respectively: compound $( \pm)-4:{ }^{1} \mathrm{H}$ NMR $(500 \mathrm{MHz}$, $\left.\mathrm{CDCl}_{3}\right) \delta 7.69-7.39(\mathrm{~m}, 10 \mathrm{H}, 2 \times \mathrm{Ar}), 4.47(\mathrm{~d}, 1 \mathrm{H}, J=5.5$ $\mathrm{Hz}, 4-\mathrm{H}), 4.38-4.24$ (m, $\left.2 \mathrm{H}, \mathrm{OCH}_{2} \mathrm{CH}_{3}\right), 2.21$ (ddd, $1 \mathrm{H}, J=$ 2.0, 5.0, $19.0 \mathrm{~Hz}, 3-\mathrm{CHH}), 2.05$ (d, $1 \mathrm{H}, J=19.0 \mathrm{~Hz}, 3-$ $\mathrm{CH} H), 1.82(\mathrm{dd}, 1 \mathrm{H}, J=2.0,5.5 \mathrm{~Hz}, 6-\mathrm{CHH}), 1.41$ (s, $3 \mathrm{H}$, 
5- $\left.\mathrm{CH}_{3}\right), 1.35$ (t, $\left.3 \mathrm{H}, J=7.0 \mathrm{~Hz}, \mathrm{OCH}_{2} \mathrm{CH}_{3}\right), 1.21(\mathrm{~d}, 1 \mathrm{H}, J$ $=5.5 \mathrm{~Hz}, 6-\mathrm{CH} H), 1.10(\mathrm{~s}, 9 \mathrm{H}$, tert-butyl $) ;{ }^{13} \mathrm{C} \mathrm{NMR}(100$ $\left.\mathrm{MHz}, \mathrm{CDCl}_{3}\right) \delta 205.43,167.05,135.75,133.33,132.94$, $129.86,127.68,71.61,61.15,45.41,44.22,43.83,26.83$, $24.75,19.29,14.27,13.75$; LRMS (EI) $m / z: 379(\mathrm{M}-t-\mathrm{Bu})^{+}$; Anal. Calcd for $\mathrm{C}_{26} \mathrm{H}_{32} \mathrm{O}_{4} \mathrm{Si}$ : C, 71.52; H, 7.39. Found: C, 71.58; H, 7.75\%; compound ( \pm )-5: ${ }^{1} \mathrm{H}$ NMR $(500 \mathrm{MHz}$, $\left.\mathrm{CDCl}_{3}\right) \delta 7.71-7.39(\mathrm{~m}, 10 \mathrm{H}, 2 \times \mathrm{Ar}), 4.41(\mathrm{t}, 1 \mathrm{H}, J=7.8$ $\mathrm{Hz}, 4-\mathrm{H}), 4.22-4.16\left(\mathrm{~m}, 2 \mathrm{H}, \mathrm{OCH}_{2} \mathrm{CH}_{3}\right), 2.20$ (dd, $1 \mathrm{H}, J=$ $8.5,18.0 \mathrm{~Hz}, 3-\mathrm{CHH}), 2.14$ (dd, $1 \mathrm{H}, J=7.5,18.0 \mathrm{~Hz}, 3-$ $\mathrm{CH} H), 1.95(\mathrm{~d}, 1 \mathrm{H}, J=5.0 \mathrm{~Hz}, 6-\mathrm{CH}), 1.85(\mathrm{~d}, 1 \mathrm{H}, J=$ $5.0 \mathrm{~Hz}, 6-\mathrm{CH} H), 1.26\left(\mathrm{~s}, 3 \mathrm{H}, 5-\mathrm{CH}_{3}\right), 1.25(\mathrm{t}, 3 \mathrm{H}, J=6.0$ $\left.\mathrm{Hz}, \mathrm{OCH}_{2} \mathrm{CH}_{3}\right), 1.11$ (s, $9 \mathrm{H}$, tert-butyl); ${ }^{13} \mathrm{C}$ NMR (100 $\left.\mathrm{MHz}, \mathrm{CDCl}_{3}\right) \delta 203.65,166.73,136.02,135.97,133.51$, $133.42,128.04,72.39,61.58,47.00,44.58,43.10,27.14$, 23.72, 19.49, 15.83, 14.45; LRMS (EI) $m / z: 379(\mathrm{M}-t-\mathrm{Bu})^{+}$; Anal. Calcd for $\mathrm{C}_{26} \mathrm{H}_{32} \mathrm{O}_{4} \mathrm{Si}$ : C, 71.52; H, 7.39. Found: C, $71.32 ; \mathrm{H}, 7.74 \%$.

$( \pm)-(r e l)-(1 S, 2 R, 4 R, 5 S)$-Ethyl 4-[(tert-butyldiphenylsilyl)oxy]-2-hydroxy-5-methyl-2-vinylbicyclo[3.1.0] hexane1-carboxylate $(( \pm)-8)$. To a stirred solution of bicyclic compound $( \pm)-7(1.6 \mathrm{~g}, 3.67 \mathrm{mmol})$ in THF $(38 \mathrm{~mL})$ was added dropwise vinylmagnesium bromide $(4.03 \mathrm{~mL}, 4.04$ mmol, $1.0 \mathrm{M}$ solution in THF) at $-78^{\circ} \mathrm{C}$ and the reaction mixture was stirred at $-78^{\circ} \mathrm{C}$ for $30 \mathrm{~min}$. Following quenching with saturated $\mathrm{NH}_{4} \mathrm{Cl}(5 \mathrm{~mL})$ at $-78^{\circ} \mathrm{C}$, the reaction mixture was extracted by ethyl acetate $(80 \mathrm{~mL} \times 2)$, dried over $\mathrm{MgSO}_{4}$, filtered, and evaporated under reduced pressure to give an oil, which was purified by silica gel column chromatography using hexane and ethyl acetate $(10: 1)$ as the eluent to give $( \pm)-8(1.38 \mathrm{~g}, 81 \%)$ as a colorless oil: ${ }^{1} \mathrm{H}$ NMR $\left(500 \mathrm{MHz}, \mathrm{CDCl}_{3}\right) \delta 7.71-7.36(\mathrm{~m}, 10 \mathrm{H}, 2 \times \mathrm{Ar}), 5.84(\mathrm{dd}$, $\left.1 \mathrm{H}, J=10.5,17.0 \mathrm{~Hz}, \mathrm{C} H=\mathrm{CH}_{2}\right), 4.95(\mathrm{~d}, 1 \mathrm{H}, J=17.0 \mathrm{~Hz}$, $\mathrm{CH}=\mathrm{C} H \mathrm{H}), 4.91(\mathrm{~d}, 1 \mathrm{H}, J=10.5 \mathrm{~Hz}, \mathrm{CH}=\mathrm{CH} H), 4.15-4.08$ (m, $\left.2 \mathrm{H}, \mathrm{OCH}_{2} \mathrm{CH}_{3}\right), 4.04$ (t, $\left.1 \mathrm{H}, J=8.0 \mathrm{~Hz}, 4-\mathrm{H}\right), 2.88(\mathrm{br}$ s, $1 \mathrm{H}, \mathrm{OH}), 1.80(\mathrm{~d}, 1 \mathrm{H}, J=5.0 \mathrm{~Hz}, 6-\mathrm{CH}), 1.62(\mathrm{dd}, 1 \mathrm{H}$, $J=7.0,13.0 \mathrm{~Hz}, 3-\mathrm{C} H \mathrm{H}), 1.52(\mathrm{dd}, 1 \mathrm{H}, J=9.0,13.0 \mathrm{~Hz}, 3-$ $\mathrm{CH} H), 1.39\left(\mathrm{~s}, 3 \mathrm{H}, 5-\mathrm{CH}_{3}\right), 1.22(\mathrm{t}, 3 \mathrm{H}, J=7.0 \mathrm{~Hz}$, $\left.\mathrm{OCH}_{2} \mathrm{CH}_{3}\right), 1.09(\mathrm{~s}, 9 \mathrm{H}$, tert-butyl), $1.03(\mathrm{~d}, 1 \mathrm{H}, J=5.0 \mathrm{~Hz}$, 6-CHH); ${ }^{13} \mathrm{C}$ NMR $\left(100 \mathrm{MHz}, \mathrm{CDCl}_{3}\right) \delta 172.71,144.40$, $136.10,136.04,134.17,133.99,129.95,127.79,111.57,77.92$, 75.04, 60.52, 41.58, 39.99, 38.33, 27.17, 20.34, 19.51, 15.78, 14.32; LRMS $(\mathrm{FAB}+) \mathrm{m} / \mathrm{z} 487(\mathrm{M}+\mathrm{Na})^{+}$; HRMS $(\mathrm{FAB}+)$ $m / z \mathrm{C}_{28} \mathrm{H}_{36} \mathrm{NaO}_{4} \mathrm{Si}(\mathrm{M}+\mathrm{Na})^{+}$calcd 487.2281, obsd 487.2264.

$( \pm)-(r e l)-(1 R, 2 R, 4 R, 5 S)-4-[($ tert-Butyldiphenylsilyl)oxy]1-hydroxymethyl-5-methyl-2-vinylbicyclo[3.1.0] hexan-2ol ((土)-9). A stirred suspension of LAH $(169 \mathrm{mg}, 4.45$ $\mathrm{mmol})$ in diethyl ether $(38 \mathrm{~mL})$ was treated dropwise with a solution of crude $( \pm)-8(1.38 \mathrm{~g}, 2.97 \mathrm{mmol})$ in diethyl ether $(7 \mathrm{~mL})$ at $0{ }^{\circ} \mathrm{C}$. After stirring for $1.5 \mathrm{~h}$, water $(0.28 \mathrm{~mL}), 15 \%$ $\mathrm{NaOH}(0.28 \mathrm{~mL})$, and water $(0.84 \mathrm{~mL})$ were added dropwise at $0{ }^{\circ} \mathrm{C}$. The reaction mixture was filtered through a pad of Celite and the filtrate was concentrated under reduced pressure. The resulting residue was purified by silica gel column chromatography using hexane and ethyl acetate (3:1) as the eluent to give diol compound $( \pm)-9(1.21 \mathrm{~g}, 96 \%)$ as a colorless oil: ${ }^{1} \mathrm{H}$ NMR $\left(500 \mathrm{MHz}, \mathrm{CDCl}_{3}\right) \delta 7.72-7.36(\mathrm{~m}$,
$10 \mathrm{H}, 2 \times \mathrm{Ar}), 5.70\left(\mathrm{dd}, 1 \mathrm{H}, J=10.5,17.0 \mathrm{~Hz}, \mathrm{CH}=\mathrm{CH}_{2}\right)$, $5.23(\mathrm{~d}, 1 \mathrm{H}, J=17.0 \mathrm{~Hz}, \mathrm{CH}=\mathrm{C} H \mathrm{H}), 5.05(\mathrm{~d}, 1 \mathrm{H}, J=10.5$ $\mathrm{Hz}, \mathrm{CH}=\mathrm{CH} H), 4.10(\mathrm{t}, 1 \mathrm{H}, J=8.0 \mathrm{~Hz}, 4-\mathrm{H}), 4.00(\mathrm{~d}, 1 \mathrm{H}, J$ $=12.0 \mathrm{~Hz}, \mathrm{CHHOH}), 3.24(\mathrm{~d}, 1 \mathrm{H}, J=12.0 \mathrm{~Hz}, \mathrm{CH} H \mathrm{OH})$, 2.49 (br s, $1 \mathrm{H}, \mathrm{OH}), 1.88$ (br s, $1 \mathrm{H}, \mathrm{OH}), 1.69$ (dd, $1 \mathrm{H}, J=$ 7.5, $13.5 \mathrm{~Hz}, 3-\mathrm{CHH}), 1.49$ (dd, $1 \mathrm{H}, J=9.5,13.5 \mathrm{~Hz}, 3-$ $\mathrm{CH} H$ ), 1.47 (d, $1 \mathrm{H}, J=6.0 \mathrm{~Hz}, 6-\mathrm{C} H \mathrm{H}), 1.09$ (s, $9 \mathrm{H}$, tertbutyl), $1.04\left(\mathrm{~s}, 3 \mathrm{H}, 5-\mathrm{CH}_{3}\right), 0.46(\mathrm{~d}, 1 \mathrm{H}, J=5.5 \mathrm{~Hz}, 6-$ $\mathrm{CH} H) ;{ }^{13} \mathrm{C}$ NMR (125 MHz, $\left.\mathrm{CDCl}_{3}\right) \delta 142.77,136.16,136.10$, $134.44,134.32,129.92,129.92,127.80,127.79,112.31$, 80.69, 76.50, 64.39, 43.41, 39.37, 33.87, 27.25, 19.58, 16.35, 16.19; LRMS (FAB+) $m / z 445(\mathrm{M}+\mathrm{Na})^{+}$; HRMS (FAB+) $m / z \mathrm{C}_{26} \mathrm{H}_{34} \mathrm{NaO}_{3} \mathrm{Si}(\mathrm{M}+\mathrm{Na})^{+}$calcd 445.2175, obsd 445.2166.

( \pm )-(rel)-(1R,2R,4R,5S)-4-[(tert-Butyldiphenylsilyl)oxy]2-hydroxy-5-methyl-2-vinylbicyclo[3.1.0] hexane-1-methyl benzoate $(( \pm)-10)$. To a stirred solution of diol compound $( \pm)-9(871 \mathrm{mg}, 2.06 \mathrm{mmol})$ in pyridine $(8 \mathrm{~mL})$ was added dropwise benzoyl chloride $(0.26 \mathrm{~mL}, 2.24 \mathrm{mmol})$ at $0{ }^{\circ} \mathrm{C}$ and the reaction mixture was stirred at room temperature for $2 \mathrm{~h}$. Following quenching with water $(0.5 \mathrm{~mL})$, the solvent was removed under reduced pressure and the residue was partitioned between ethyl acetate $(100 \mathrm{~mL} \times 2)$ and water $(30 \mathrm{~mL})$. The organic layer was washed with $0.5 \mathrm{~N} \mathrm{HCl}$ and aqueous saturated $\mathrm{NaHCO}_{3}$ solution successively, dried over $\mathrm{MgSO}_{4}$ and filtered. The filtrate was concentrated under reduced pressure to give an oil, which was purified by silica gel column chromatography using hexane and ethyl acetate $(9: 1)$ as the eluent to give vinyl compound $( \pm)-10(953 \mathrm{mg}$, $88 \%)$ as a colorless oil: ${ }^{1} \mathrm{H}$ NMR $\left(400 \mathrm{MHz}, \mathrm{CDCl}_{3}\right) \delta 7.98-$ $7.33(\mathrm{~m}, 15 \mathrm{H}, 3 \times \mathrm{Ar}), 5.66(\mathrm{dd}, 1 \mathrm{H}, J=10.4,17.6 \mathrm{~Hz}$, $\left.\mathrm{C} H=\mathrm{CH}_{2}\right), 5.17(\mathrm{dd}, 1 \mathrm{H}, J=1.2,17.6 \mathrm{~Hz}, \mathrm{CH}=\mathrm{C} H \mathrm{H}), 4.98$ (dd, $1 \mathrm{H}, J=1.2,10.4 \mathrm{~Hz}, \mathrm{CH}=\mathrm{CH} H), 4.52(\mathrm{~d}, 1 \mathrm{H}, J=12.4$ $\mathrm{Hz}, \mathrm{BzOCHH}), 4.13(\mathrm{t}, 1 \mathrm{H}, J=8.0 \mathrm{~Hz}, 4-\mathrm{CH}), 4.06(\mathrm{~d}, 1 \mathrm{H}$, $J=12.0 \mathrm{~Hz}, \mathrm{BzOCH} H), 2.52(\mathrm{~s}, 1 \mathrm{H}, \mathrm{OH}), 1.72(\mathrm{dd}, 1 \mathrm{H}, J=$ 7.2, 13.2 Hz, 3-CHH), 1.52 (dd, $1 \mathrm{H}, J=8.8,13.2 \mathrm{~Hz}, 3-$ $\mathrm{CH} H), 1.50(\mathrm{~d}, 1 \mathrm{H}, J=5.6 \mathrm{~Hz}, 6-\mathrm{CH}), 1.11(\mathrm{~s}, 3 \mathrm{H}, 5-$ $\mathrm{CH}_{3}$ ), 1.06 (s, $9 \mathrm{H}$, tert-butyl), $0.54(\mathrm{~d}, 1 \mathrm{H}, J=5.6 \mathrm{~Hz}, 6-$ $\mathrm{CH} H) ;{ }^{13} \mathrm{C}$ NMR $\left(100 \mathrm{MHz}, \mathrm{CDCl}_{3}\right) \delta 167.15,142.59,136.14$, $136.08,134.34,134.25,133.32,130.26,129.94,129.75$, $128.69,127.80,112.38,80.03,76.21,65.51,43.39,37.61$, 34.84, 27.21, 19.55, 16.60, 15.99; LRMS (FAB+) $m / z 549$ $(\mathrm{M}+\mathrm{Na})^{+}$; HRMS (FAB + ) $m / z \mathrm{C}_{33} \mathrm{H}_{38} \mathrm{NaO}_{4} \mathrm{Si}(\mathrm{M}+\mathrm{Na})^{+}$calcd 549.2437, obsd 549.2424.

$( \pm)-(r e l)-(1 R, 2 R, 4 R, 5 S)-2,4-D i h y d r o x y-5-m e t h y l-2$-vinylbicyclo[3.1.0] hexane-1-methyl benzoate (( \pm )-11). A stirred solution of benzoyl compound $( \pm)-\mathbf{1 0}(936 \mathrm{mg}, 1.78 \mathrm{mmol}$ ) in THF $(7 \mathrm{~mL})$ was treated dropwise with $1 N$ TBAF $(2.1$ $\mathrm{mL}, 2.14 \mathrm{mmol}$ ) at room temperature and stirred for $1 \mathrm{~d}$. After the solvent was removed under reduced pressure, the residue was purified by silica gel column chromatography using hexane and ethyl acetate $(1: 1.7)$ as the eluent to give the glycosyl donor $( \pm)-11(460 \mathrm{mg}, 90 \%)$ as a colorless oil: ${ }^{1} \mathrm{H}$ NMR $\left(500 \mathrm{MHz}, \mathrm{CDCl}_{3}\right) \delta 8.12-7.44(\mathrm{~m}, 5 \mathrm{H}, \mathrm{Ar}), 5.92$ (dd, $\left.1 \mathrm{H}, J=10.5,17.0 \mathrm{~Hz}, \mathrm{CH}=\mathrm{CH}_{2}\right), 5.41(\mathrm{dd}, 1 \mathrm{H}, J=1.0$, $17.0 \mathrm{~Hz}, \mathrm{CH}=\mathrm{C} H \mathrm{H}), 5.17(\mathrm{dd}, 1 \mathrm{H}, J=1.0,10.5 \mathrm{~Hz}$, $\mathrm{CH}=\mathrm{CH} H), 4.65(\mathrm{~d}, 1 \mathrm{H}, J=12.5 \mathrm{~Hz}, \mathrm{BzOC} H \mathrm{H}), 4.20(\mathrm{t}, 1$ $\mathrm{H}, J=8.5 \mathrm{~Hz}, 4-\mathrm{H}), 4.17$ (d, $1 \mathrm{H}, J=12.5 \mathrm{~Hz}, \mathrm{BzOCH} H$ ), 
$2.14(\mathrm{dd}, 1 \mathrm{H}, J=7.5,13.5 \mathrm{~Hz}, 3-\mathrm{CH}), 1.50(\mathrm{dd}, 1 \mathrm{H}, J=$ 9.0, $13.5 \mathrm{~Hz}, 3-\mathrm{CH} H), 1.42(\mathrm{~d}, 1 \mathrm{H}, J=5.5 \mathrm{~Hz}, 6-\mathrm{CH})$, $1.35\left(\mathrm{~s}, 3 \mathrm{H}, 5-\mathrm{CH}_{3}\right), 0.58(\mathrm{~d}, 1 \mathrm{H}, J=5.5 \mathrm{~Hz}, 6-\mathrm{CH} H) ;{ }^{13} \mathrm{C}$ NMR $\left(125 \mathrm{MHz}, \mathrm{CDCl}_{3}\right) \delta 166.94,142.26,133.16,129.90$, $129.67,128.49,112.57,79.95,75.05,65.01,43.23,38.12$, $34.51,15.96,15.19$.

( \pm )-((rel)-(1R,2R,4S,5S)-4-(6-Chloro-9H-purin-9-yl)-2hydroxy-5-methyl-2-vinylbicyclo[3.1.0|hexane-1-yl)methyl benzoate $(( \pm)-12)$. To a stirred solution of glycosyl donor ( \pm )-11 (185 mg, $0.64 \mathrm{mmol})$, triphenylphosphine (219 mg, $0.83 \mathrm{mmol})$, and 6-chloropurine $(129 \mathrm{mg}, 0.83 \mathrm{mmol})$ in anhydrous THF $(5 \mathrm{~mL})$ was added dropwise diethyl azodicarboxylate $(0.13 \mathrm{~mL}, 0.83 \mathrm{mmol})$ at $0{ }^{\circ} \mathrm{C}$, and the reaction mixture was stirred at room temperature for $2 \mathrm{~h}$. The volatiles were evaporated in vacuo and the resulting residue was purified by silica gel column chromatography using hexane and ethyl acetate $(1: 1)$ as the eluent to give $( \pm)-\mathbf{1 2}$ $(200 \mathrm{mg}, 73 \%)$ as a colorless oil: ${ }^{1} \mathrm{H}$ NMR $(500 \mathrm{MHz}$, $\left.\mathrm{CDCl}_{3}\right) \delta 8.80(\mathrm{~s}, 1 \mathrm{H}, \mathrm{H}-8), 8.37(\mathrm{~s}, 1 \mathrm{H}, \mathrm{H}-2), 8.07-7.47$ (m, $5 \mathrm{H}, \mathrm{Ar}), 6.49$ (dd, $1 \mathrm{H}, J=10.5,17.0 \mathrm{~Hz}, \mathrm{CH}=\mathrm{CH}_{2}$ ), $5.44(\mathrm{~d}, 1 \mathrm{H}, J=8.0 \mathrm{~Hz}, 4-\mathrm{H}), 5.36(\mathrm{~d}, 1 \mathrm{H}, J=17.0 \mathrm{~Hz}$, $\mathrm{CH}=\mathrm{CHH}), 5.30(\mathrm{~d}, 1 \mathrm{H}, J=10.5 \mathrm{~Hz}, \mathrm{CH}=\mathrm{CH} H), 4.68(\mathrm{~d}, 1$ $\mathrm{H}, J=13.0 \mathrm{~Hz}, \mathrm{BzOCH \textrm {H }}), 4.63(\mathrm{~d}, 1 \mathrm{H}, J=13.0 \mathrm{~Hz}$, $\mathrm{BzOCH} H), 2.42(\mathrm{dd}, 1 \mathrm{H}, J=8.0,16.0 \mathrm{~Hz}, 3-\mathrm{CHH}), 2.22(\mathrm{~d}$, $1 \mathrm{H}, J=16.0 \mathrm{~Hz}, 3-\mathrm{CH} H), 1.44(\mathrm{~d}, 1 \mathrm{H}, J=5.5 \mathrm{~Hz}, 6-\mathrm{CHH})$, $1.17\left(\mathrm{~s}, 3 \mathrm{H}, 5-\mathrm{CH}_{3}\right), 0.91(\mathrm{~d}, 1 \mathrm{H}, J=5.5 \mathrm{~Hz}, 6-\mathrm{CH} H) ;{ }^{13} \mathrm{C}$ NMR $\left(125 \mathrm{MHz}, \mathrm{CDCl}_{3}\right) \delta 166.73,152.03,151.87,151.32$, $144.37,140.60,133.47,131.56,129.64,129.46,128.78$, $115.09,81.90,64.43,60.12,44.08,41.79,32.86,20.87,15.28$.

( \pm )-(rel)-(1R,2R,4S,5S)-4-(6-Amino-9H-purin-9-yl)-1(hydroxymethyl)-5-methyl-2-vinylbicyclo[3.1.0]hexan-2ol (( \pm$)-1)$. A solution of $( \pm)-12(61 \mathrm{mg}, 0.14 \mathrm{mmol})$ and saturated methanolic ammonia $(6 \mathrm{~mL})$ was heated in a glass bomb at $80^{\circ} \mathrm{C}$ for $12 \mathrm{~h}$. The reaction mixture was evaporated in vacuo and purified by silica gel column chromatography using methylene chloride and methanol (10:1) as the eluent to give the adenine nucleoside $( \pm)-\mathbf{1}(31 \mathrm{mg}, 72 \%)$ as a white solid: mp 104-108 ${ }^{\circ} \mathrm{C}$; UV (MeOH) $\lambda_{\max } 260.0 \mathrm{~nm} ;{ }^{1} \mathrm{H}$ NMR (500 MHz, methanol- $\left.d_{4}\right) \delta 8.49$ (s, $\left.1 \mathrm{H}, \mathrm{H}-8\right), 8.23$ (s, $1 \mathrm{H}$, $\mathrm{H}-2), 6.51\left(\mathrm{dd}, 1 \mathrm{H}, J=10.5,17.0 \mathrm{~Hz}, \mathrm{CH}=\mathrm{CH}_{2}\right), 5.19$ (d, 1 $\mathrm{H}, J=7.5 \mathrm{~Hz}, 4-\mathrm{H}), 5.06(\mathrm{~d}, 1 \mathrm{H}, J=10.5 \mathrm{~Hz}, \mathrm{CH}=\mathrm{CHH})$, $5.05(\mathrm{~d}, 1 \mathrm{H}, J=17.0 \mathrm{~Hz}, \mathrm{CH}=\mathrm{CH} H), 4.23(\mathrm{~d}, 1 \mathrm{H}, J=12.0$ $\mathrm{Hz}, \mathrm{CHHOH}), 3.64$ (d, $1 \mathrm{H}, J=12.0 \mathrm{~Hz}, \mathrm{CH} H \mathrm{OH}), 2.17$ (dd, $1 \mathrm{H}, J=7.5,15.0 \mathrm{~Hz}, 3-\mathrm{CHH}), 2.03$ (d, $1 \mathrm{H}, J=15.0$ $\mathrm{Hz}, 3-\mathrm{CH} H), 1.28$ (d, $1 \mathrm{H}, J=5.5 \mathrm{~Hz}, 6-\mathrm{CHH}), 1.22$ (s, $3 \mathrm{H}$, $\left.5-\mathrm{CH}_{3}\right), 0.70(\mathrm{~d}, 1 \mathrm{H}, J=5.5 \mathrm{~Hz}, 6-\mathrm{CH} H) ;{ }^{13} \mathrm{C} \mathrm{NMR}(125$ $\left.\mathrm{MHz}, \mathrm{MeOH}-d_{4}\right) \delta 156.17,152.46,149.35,141.77,141.49$, $118.69,112.08,81.66,61.27,60.29,43.98,43.41,30.73$, 20.42, 14.83; LRMS (FAB+) $m / z 302(\mathrm{M}+\mathrm{H})^{+}$; HRMS (FAB + ) $m / z \mathrm{C}_{15} \mathrm{H}_{20} \mathrm{~N}_{5} \mathrm{O}_{2}(\mathrm{M}+\mathrm{H})^{+}$calcd 302.1617, obsd 302.1613.

( \pm )-9-[(rel)-(1S,2S,4R,5R)-4-Hydroxy-5-(hydroxymethyl)-1-methyl-4-vinylbicyclo[3.1.0]hexan-2-yl]-9H-purin6-ol (( \pm$)-2)$. To a stirred solution of $( \pm)-12(62 \mathrm{mg}, 0.14$ $\mathrm{mmol})$ in methanol $(3 \mathrm{~mL})$ were added 2-mercaptoethanol (0.06 $\mathrm{mL}, 0.87 \mathrm{mmol})$ and $1 N \mathrm{NaOMe}(0.87 \mathrm{~mL}, 0.87$ $\mathrm{mmol}$ ) and the mixture was refluxed for $3 \mathrm{~h}$. After cooling, the reaction mixture was evaporated in vacuo, and the residue was purified by silica gel column chromatography using methylene chloride and methanol (10:1) as the eluent to give the hypoxanthine nucleoside $( \pm)-2(33 \mathrm{mg}, 75 \%)$ as a white solid: mp 124-132 ${ }^{\circ} \mathrm{C}$; UV (MeOH) $\lambda_{\max } 249.0 \mathrm{~nm} ;{ }^{1} \mathrm{H}$ NMR (500 MHz, methanol- $\left.d_{4}\right) \delta 8.46$ (s, $\left.1 \mathrm{H}, \mathrm{H}-8\right), 8.09$ (s, $1 \mathrm{H}, \mathrm{H}-2), 6.50$ (dd, $\left.1 \mathrm{H}, J=10.5,17.0 \mathrm{~Hz}, \mathrm{CH}=\mathrm{CH}_{2}\right), 5.22$ (d, $1 \mathrm{H}, J=7.0 \mathrm{~Hz}, 2-\mathrm{H}), 5.08(\mathrm{~d}, 1 \mathrm{H}, J=10.5 \mathrm{~Hz}, \mathrm{CH}=$ $\mathrm{CHH}), 5.06(\mathrm{~d}, 1 \mathrm{H}, J=17.0 \mathrm{~Hz}, \mathrm{CH}=\mathrm{CH} H), 4.20(\mathrm{~d}, 1 \mathrm{H}, J$ $=12.0 \mathrm{~Hz}, \mathrm{CHHOH}), 3.65(\mathrm{~d}, 1 \mathrm{H}, J=12.0 \mathrm{~Hz}, \mathrm{CH} H \mathrm{OH})$, $2.17(\mathrm{dd}, 1 \mathrm{H}, J=7.0,16.0 \mathrm{~Hz}, 3-\mathrm{CHH}), 2.06(\mathrm{~d}, 1 \mathrm{H}, J=$ $16.0 \mathrm{~Hz}, 3-\mathrm{CH} H), 1.27$ (d, $1 \mathrm{H}, J=5.5 \mathrm{~Hz}, 6-\mathrm{CHH}), 1.21$ (s, $\left.3 \mathrm{H}, 1-\mathrm{CH}_{3}\right), 0.70(\mathrm{~d}, 1 \mathrm{H}, J=5.5 \mathrm{~Hz}, 6-\mathrm{CH} H) ;{ }^{13} \mathrm{C} \mathrm{NMR}$ (125 MHz, methanol- $\left.d_{4}\right) \delta 157.82,148.92,145.42,141.66$, $140.86,123.71,112.24,81.65,61.10,60.41,43.91,43.29$, 30.78, 20.27, 14.71; LRMS (FAB+) m/z $302(\mathrm{M})^{+}, 303$ $(\mathrm{M}+\mathrm{H})^{+}, 325(\mathrm{M}+\mathrm{Na})^{+}$; HRMS (FAB+) $m / z \mathrm{C}_{15} \mathrm{H}_{19} \mathrm{~N}_{4} \mathrm{O}_{3}$ $(\mathrm{M}+\mathrm{H})^{+}$calcd 303.1457, obsd 303.1465.

\section{Results and Discussion}

A strategy for the synthesis of 5-methylbicyclo[3.1.0]hexanone template, $( \pm)-7$, is described in Scheme 1. It was envisioned that a diazo compound $( \pm)-5$ could be an appropriate intermediate to synthesize 5-methylbicyclo[3.1.0]hexanone $( \pm)-7$ and its epimer $( \pm)-6$, the former of which could be converted into bicyclo[3.1.0]hexyl carbanucleosides with a $\beta$-3'-ethenyl group.

Reaction of methacrolein with ethyl acetoacetate dianion obtained from treating with 2 equiv. LDA produced unstable $\delta$-hydroxy- $\beta$-keto ethyl ester ( \pm )-3 via an 1,2-addition reaction $^{2}$ (Scheme 1). After silylation of the resulting hydroxyl group, diazotization with tosyl azide ${ }^{3}$ in the presence of triethylamine produced diazo compound $( \pm)-5$ in a quantitative yield, which could be utilized as a substrate of a carbenoid

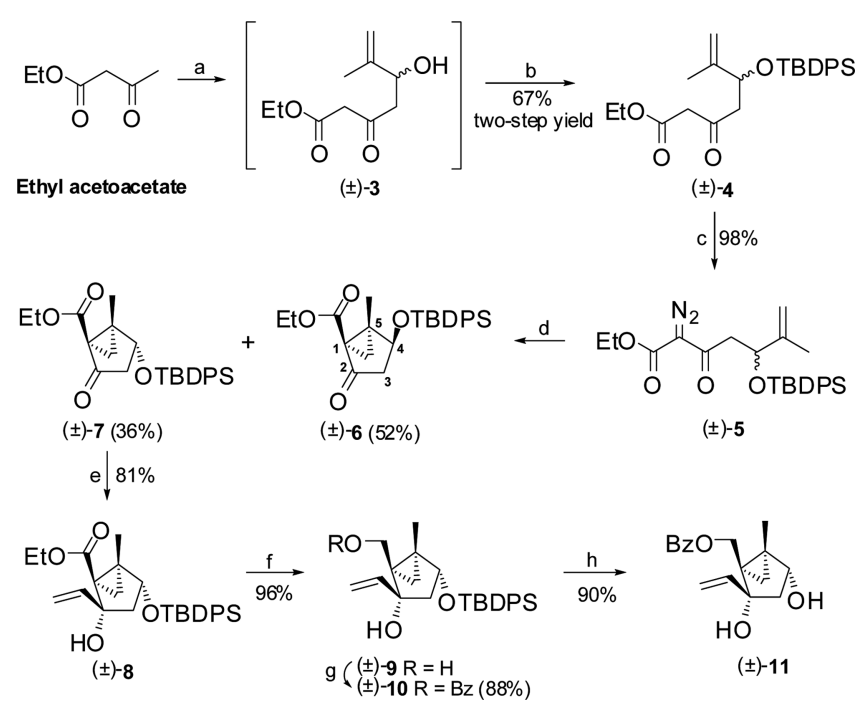

Scheme 1. Reagents and conditions: (a) (i) LDA, THF, $0{ }^{\circ} \mathrm{C}$; (ii) methacrolein; (b) TBDPSCl, imidazole, $\mathrm{CH}_{2} \mathrm{Cl}_{2}, \mathrm{rt}, 6$ h; (c) $\mathrm{TsN}_{3}$, $\mathrm{Et}_{3} \mathrm{~N}, \mathrm{CH}_{3} \mathrm{CN}$, rt, $21 \mathrm{~h}$; (d) $\mathrm{Rh}_{2}(\mathrm{OAc})_{4}$, benzene, rt, $24 \mathrm{~h}$; (e) $\mathrm{CH}_{2}=\mathrm{CHMgBr}$, THF, $-78^{\circ} \mathrm{C}, 30 \mathrm{~min}$; (f) $\mathrm{LiAlH}_{4}$, ether, $0{ }^{\circ} \mathrm{C}, 1.5$ h; (g) BzCl, pyridine, rt, 2 h; (h) TBAF, THF, rt, 16 h. 
reaction. A skeleton of 5-methylbicyclo[3.1.0]hexanone ( \pm -) 7 , the key intermediate, could be easily synthesized from a carbene insertion reaction towards a carbon-carbon double bond. Several metal catalysts were employed for the carbenoid cycloaddition. ${ }^{8,14,15}$ Out of them, rhodium (II) acetate dimer ${ }^{15}$ revealed the best results. Although rhodium (II) acetate dimer gave a little higher ratio of the undesired 5methylbicyclo[3.1.0]hexanone $( \pm)-6$ over the desired one, $( \pm)-7(( \pm)-6:( \pm)-7=52 \%: 36 \%)$, a smaller amount of byproducts was generated and the yield also was improved upon using rhodium (II) acetate dimer as a catalyst of carbenoid cycloaddition. The reaction smoothly proceeded at room temperature. Assignment of these diastereomers was unambiguously confirmed from peak splitting pattern of each anomeric proton in their ${ }^{1} \mathrm{H}$ NMR spectra. The anomeric proton signal of $( \pm)-\mathbf{6}$ appeared as a doublet whereas that of $( \pm)-7$ appeared as a triplet. ${ }^{16}$ In the rigid bicyclo[3.1.0]hexanone template of $( \pm)-6$, the dihedral angle between $\mathrm{H}_{4}$ (an anomeric hydrogen) and $\mathrm{H}_{3 \text {-exo }}$ is near $90^{\circ}$, indicating that its anomeric proton signal appears as a doublet. The desired bicyclo[3.1.0]hexanone template $( \pm)-7$ obtained via four steps had a correct relative stereochemistry of three chiral carbons as well as substituents at appropriate positions. Introduction of a vinyl group into C2-up position is depicted in Scheme 1. A Grignard reaction of the obtained bicyclo[3.1.0]hexanone ( \pm )-7 with vinylmagnesium bromide ${ }^{17}$ proceeded to produce $\alpha$-tertiary allylic alcohols $( \pm)-\mathbf{8}$ as a single stereoisomer in $81 \%$ yield. The ethenide attack occurred with remarkably high diastereoselectivity from the less encumbered convex face ( $\beta$-face) of the bicyclo[3.1.0]hexanone system. In addition, the Grignard reaction chemoselectively proceeded: the reaction occurred only at the ketone functionality in the presence of an ester functional group. The stereochemistry of the new generated asymmetric center was determined with compound $( \pm)$-10 by NOE experiments. $\mathrm{LiAlH}_{4}$ was used for the reduction of the ester group of $( \pm)-\mathbf{8}$ to produce diol, $( \pm)-\mathbf{9}$. Benzoylation of $( \pm)-9$ by treatment with benzoyl chloride and pyridine at room temperature gave monobenzoate $( \pm)-\mathbf{1 0}$ as a major product along with dibenzoate as a minor product, maybe due to the steric hindrance of the tertiary allylic alcohol. We thought that the tert-hydroxyl group in $( \pm)$-10 wouldn't participate in a Mitsunobu reaction ${ }^{18}$ for the condensation with nucleobases even if it does not be protected. Therefore, monobenzoate $( \pm)$-10 obtained as a major product was considered a useful substrate for the synthesis of the desired glycosyl

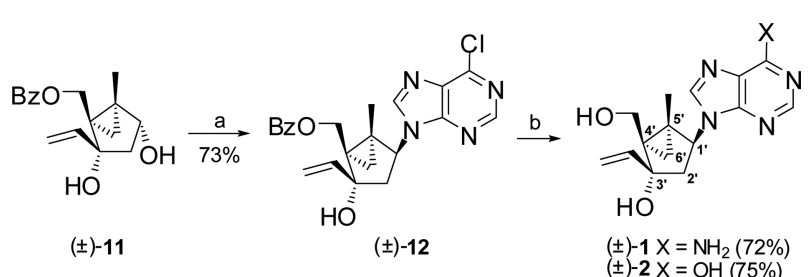

Scheme 2. Reagents and conditions: (a) 6-chloropurine, $\mathrm{PPh}_{3}$, DEAD, THF, rt, 2 h; (b) $\mathrm{NH}_{3} / \mathrm{MeOH}, 80{ }^{\circ} \mathrm{C}, 12 \mathrm{~h}$ for $( \pm)-\mathbf{1}$; $1 \mathrm{~N}-$ $\mathrm{NaOMe}, 2$-mercaptoethanol, $\mathrm{MeOH}$, reflux, $3 \mathrm{~h}$ for $( \pm)-2$. donor $( \pm)$-11. Desilylation with a fluoride source gave the glycosyl donor $( \pm)-\mathbf{1 1}$.

Synthesis of adenine and hypoxanthine bicyclo[3.1.0]hexyl carbanucleosides with a $\beta-3^{\prime}$-ethenyl group was shown in Scheme 2. Coupling of the glycosyl donor ( \pm )-11 with 6chloropurine nucleobase was successfully accomplished under Mitsunobu conditions using $\mathrm{PPh}_{3}$ and DEAD. As we expected, compound $( \pm)$-11 with both secondary and tertiary hydroxyl groups served as a good glycosyl donor during the Mitsunobu reaction. The desired $N^{9}$-alkylated nucleoside $( \pm)$-12 was generated as a single diastereomer without the formation of its $N^{7}$-regioisomer. Treatment of 6-chloropurine bicyclo[3.1.0]hexyl carbanucleoside ( \pm )-12 with methanolic ammonia afforded the desired adenine carbanucleoside $( \pm)-\mathbf{1}$ via amination at the chloro position and debenzoylation and treatment with $1 \mathrm{~N}-\mathrm{NaOMe}$ and 2-mercaptoethanol produced the desired hypoxanthine carbanucleoside $( \pm)$-2 via hydroxylation at the chloro position and debenzoylation. According to literature $\mathrm{UV}$ data, $N^{9}$ - and $N^{7}$-adenine nucleosides revealed their $\lambda_{\max }$ values at 260 and $272 \mathrm{~nm}$, respectively. ${ }^{19,20}$ Observation of UV (MeOH) $\lambda_{\max } 260.0 \mathrm{~nm}$ in adenine nucleoside, $( \pm)$-1, confirmed that $( \pm)$-12 was a desired $N^{9}-$ regioisomer. Since each of methylene protons attached to $\mathrm{C} 2^{\prime}$ and $\mathrm{C}^{\prime}$ of $5^{\prime}$-methylbicyclo[3.1.0] hexyl carbanucleosides was chemically nonequivalent and the bicyclo[3.1.0]hexyl template was conformationally fixed and compact, chemical shifts of the nonequivalent protons on the same carbons might be affected in a very different degree by the anisotropic effect of neighboring substituents such as the ethenyl group and nucleobase. The differences of chemical shifts of the protons attached to $\mathrm{C} 2^{\prime}$ and $\mathrm{C} 6^{\prime}$ were in a variety of range. Each anomeric proton (located in a carbon bearing nucleobases) signal of two final nucleosides appeared as a doublet, indicating that both nucleosides, $( \pm)-\mathbf{1}$ and $( \pm)-\mathbf{2}$, accommodate the north conformation and were $\beta$-anomers.

Antiviral activities of the adenine and hypoxanthine nucleosides, $( \pm)$-1 and $( \pm)$-2 were evaluated against EMCV, Coxackie B3 virus, VSV, influenza viruses (Seoul, Taiwan, and Panama), HSV-1 and 2, and HIV-1 and 2. The final compounds showed neither antiviral activity nor cytotoxicity up to $100 \mu \mathrm{g} / \mathrm{mL}$, except for adenine nucleoside $( \pm)-\mathbf{1}$ showing a cytotoxicity-induced, moderate anti-HIV activity in MT-4 cell lines.

The reason why these nucleosides did not show antiviral activity might be attributed to steric hindrance of the $5^{\prime}$ methyl group upon the access of kinases associated with nucleoside metabolism or the unfavored glycosyl torsion angle $\chi^{21}$ (which determines the syn or anti disposition of the nucleobase relative to the sugar moiety) for phosphorylation by kinases due to steric hindrance between the 5 '-methyl group and the nucleobase. Therefore, a synthetic method for bicyclo[3.1.0]hexyl nucleosides devoid of the $5^{\prime}$-methyl group is being designed.

\section{Conclusion}

Conformationally fixed bicyclo[3.1.0]hexyl purine nucleo- 
sides with a $\beta$-ethenyl group at C3'-position were successfully synthesized as potential anticancer and antiviral agents starting from simple materials, ethyl acetoacetate and methacrolein. Intramolecular carbenoid cycloaddition and a diastereo- and chemo-selective Grignard reaction were utilized as the key steps. It is of great interest to note that an intramolecular carbenoid cycloaddition of $( \pm)-5$ afforded a bicyclo[3.1.0]hexanone scaffold ( \pm )-7 bearing an appropriate relative stereochemistry of three chiral centers generated during this reaction. Compound $( \pm)$-11 with both secondary and tertiary hydroxyl groups was proved to act as a good substrate for a Mitsunobu reaction.

Acknowledgments. This work was supported for two years by Pusan national University Research Grant.

\section{References}

1. Borthwick, A. D.; Biggadike, K. Tetrahedron 1992, 48, 571.

2. Crimmins, M. T. Tetrahedron 1998, 54, 9229.

3. Zhu, X.-F. Nucleos. Nucleot. Nucleic Acids 2000, 19, 651.

4. Saenger, W. In Principles of Nucleic Acid Structure; SpringerVerlag: New York, 1984; pp 51-104.

5. Plavec, J.; Tong, W.; Chattopadhyaya, J. J. Am. Chem. Soc. 1993, $115,9734$.

6. Altona, C.; Sundaralingam, M. J. Am. Chem. Soc. 1972, 94, 8205.

7. Altmann, K.-H.; Kesselring, R.; Francotte, E.; Rihs, G. Tetrahedron Lett. 1994, 35, 2331.
8. Shin, K. J.; Moon, H. R.; George, C.; Marquez, V. E. J. Org Chem. 2000, 65, 2172.

9. Lee, J. A.; Moon, H. R.; Kim, H. O.; Kim, K. R.; Lee, K. M.; Kim, B. T.; Hwang, K. J.; Chun, M. W.; Jacobson, K. A.; Jeong, L. S. J. Org. Chem. 2005, 70, 5006.

10. Park, A.-Y.; Moon, H. R.; Kim, K. R.; Chun, M. W.; Jeong, L. S. Org. Biomol. Chem. 2006, 4, 4065.

11. Choi, W. J.; Ko, Y. J.; Chandra, G.; Lee, H. W.; Kim, H. O.; Koh, H. J.; Moon, H. R.; Jung, Y. H.; Jeong, L. S. Tetrahedron 2012, $68,1253$.

12. Matsuda, A.; Hattori, H.; Tanaka, M.; Sasaki, T. Bioorg. Med. Chem. Lett. 1996, 6, 1887.

13. Matsuda, A.; Sasaki, T. Cancer Sci. 2004, 95, 105.

14. Moon, H. R.; Ford, H., Jr.; Marquez, V. E. Org. Lett. 2000, 2, 3793.

15. England, D. B.; Eagan, J. M.; Merey, G.; Anac, O.; Padwa, A. Tetrahedron 2008, 64, 988.

16. Choi, Y.; George, C.; Comin, M. J.; Barchi, J. J., Jr.; Kim, H. S.; Jacobson, K. A.; Balzarini, J.; Mitsuya, H.; Boyer, P. L.; Hughes, S. H.; Marquez, V. E. J. Med. Chem. 2003, 46, 3292.

17. Chen, X.; Zhou, W.; Schinazi, R. F.; Chu, C. K. J. Org. Chem. 2004, 69, 6034 .

18. Mitsunobu, O. Synthesis 1981, 1.

19. Panzica, R. P.; Rousseau, R. J.; Robins, R. K.; Townsend, L. B. J. Am. Chem. Soc. 1972, 94, 4708.

20. Rousseau, R. J.; Robins, R. K.; Townsend, L. B. J. Am. Chem. Soc. 1968, 90, 2661.

21. For a compressive review of this concept: Saenger, W. The Principles of Nucleic Acid Structure; Springer-Verlag: New York, 1984. 